\title{
Fluvoxamine Inhibits Weight Gain and Food Intake in Food Restricted Hyperphagic Wistar Rats
}

\author{
Tomokazu Shinozaki, Masaki Kimura, * Makoto Hosoyamada, and Toshiaki SHibasaki \\ Division of Pharmacotherapeutics, Faculty of Pharmacy, Keio University; 1-5-30 Shibakoen, Minato-ku, Tokyo 105-8512, \\ Japan. Received June 30, 2008; accepted September 10, 2008; published online September 16, 2008
}

The effects of fluvoxamine, a selective serotonin reuptake inhibitor (SSRI), were studied in normophagic and food-restriction-induced hyperphagic middle-aged Wistar rats. Normophagic intact Wistar rats were given fluvoxamine $(100 \mathrm{mg} / \mathrm{kg} / \mathrm{d}$, per os (p.o.)) or vehicle for $10 \mathrm{~d}$. Hyperphagic middle-aged Wistar rats were subjected to $10 \mathrm{~d}$ of food restriction; they were allowed to refeed for $10 \mathrm{~d}$, with ad libitum food access and administered fluvoxamine $(100 \mathrm{mg} / \mathrm{kg} / \mathrm{d}$, p.o. $)$ or vehicle during the 10 -d refeeding period. Fluvoxamine administration to normophagic middle-aged Wistar rats affected neither their weight nor food intake. However, administration to food-restricted rats showed inhibitory effects of weight gain and food intake during $10 \mathrm{~d}$ of refeeding. Fluvoxamine-treated rats showed significantly lower neuropeptide Y (NPY) immunostaining levels in the paraventricular nucleus (PVN) and dorsomedial hypothalamic nucleus (DMH) than untreated controls. Hypophagic and weightinhibiting effects of fluvoxamine might be mediated via decreased NPY in PVN and DMH. These results suggest that the appetite-controlling effect of fluvoxamine might be responsive to the rats' appetite condition.

Key words neuropeptide Y; fluvoxamine; 5-hydroxytryptamine; obesity; appetite condition

Obesity is an important factor of metabolic syndrome. It is closely related to diabetes, hypertension, and hyperlipemia. ${ }^{1)}$ The population of obese people increases daily; obesity is expected to become an increasingly serious social problem worldwide. For those reasons, immediate study of obesity treatments is necessary.

Many researchers have sought to provide methods of weight loss for treating obesity, but no reliable evidence of such a method providing long-term weight loss or prevention of weight gain has been forthcoming. Several therapeutic drugs and chemicals for treating obesity have been reported throughout the world, ${ }^{2,3)}$ and have been used as one option to control rebound after weight loss by patients who do not attain weight loss through therapeutic exercise and food restriction. Reportedly, some drugs influence the brain and thereby inhibit appetite. Although some people who take these drugs lose weight and avoid subsequent weight gain, others are apparently unaffected. For reasons that remain unclear, some people can not be treated.

The hypothalamus plays an important role in the control of food intake and body weight. ${ }^{4,5)}$ Serotonin (5-HT: 5-hydroxtryptamine) is known as an anorexigenic neurotransmitter that modulates the energy balance. Therefore, serotonergic drugs were treated for obesity until 1990's, 6,7) and sibutramine, serotonin noradrenaline reuptake inhibitor, is used to improve overweight conditions by reducing food consumption. ${ }^{899}$ Mainly, 5-HT is synthesized in the dorsal raphe nucleus (DRN), which projects to the hypothalamus. ${ }^{10)}$ The existence of 5-HT and its receptors are reported in the hypothalamic arcuate nucleus (ARC) ${ }^{11,12)}$ and the dorsomedial hypothalamic nucleus (DMH) ${ }^{13)}$ which are inferred to be important appetite control regions under the administration of serotonin selective reuptake inhibitor (SSRI). Nevertheless, conflicting data related to the appetite control effect of SSRI have been reported; moreover, the weight loss mechanism of SSRI remains unclear. A recent report described appetite controls of SSRI for young rats and obese rats, ${ }^{14-16)}$ but the effect of SSRI on middle-aged rats has not yet been investigated.
Neuropeptide Y (NPY), which is synthesized in the ARC and $\mathrm{DMH},{ }^{17,18)}$ is known as a strong feeding peptide. The NPY neurons at the ARC and DMH project into the paraventricular nucleus (PVN) and elicit feeding behavior. ${ }^{19,20)}$ Actually, NPY induces hyperphagia when the NPY peptide is administered into the PVN. ${ }^{21)}$ A negative energy balance, such as food restriction, putatively stimulates the NPY-ergic ARC to PVN and DMH to PVN pathways. ${ }^{22,23)}$

This study is designed to show: (i) whether fluvoxamine administration to normophagic middle-aged Wistar rats engenders a reduction of body weight and food intake; and (ii) whether fluvoxamine administration to food-restricted hyperphagic middle-aged Wistar rats engenders inhibition of weight gain and food intake. Furthermore, we examined (iii) whether changes in NPY-ergic projection are involved in the mechanism.

\section{MATERIALS AND METHODS}

Animals In all, 22 male Wistar rats $(404 \pm 19 \mathrm{~g}$, mean \pm S.E. mean, about 30 weeks, retired; Sankyo Labo Service Co., Tokyo) were used for Experiment $1(n=10)$ and Experiment $2(n=12)$. The rats were housed in individual cages under controlled temperatures of $23.8 \pm 0.4^{\circ} \mathrm{C}$ and were exposed to a $12: 12$-h light-dark cycle (lights on at 8:00, off at 20:00). They were provided ad libitum access to water and standard chow food (CE-2; CLEA Japan Inc., Tokyo). All care and procedures in this study were based on the principles and guidelines of Keio University.

Experiment 1: Fluvoxamine Administration to ad libitum Control Rats After 7 days' acclimation, the control group rats were separated randomly into two groups $(n=5$ per group). One group of five rats was administered fluvoxamine maleate (Meiji Seika Kaisha Ltd., Tokyo); the other was administered control vehicle. Fluvoxamine maleate was dissolved in deionized water; then administered orally $100 \mathrm{mg} / \mathrm{kg} / \mathrm{d}$ at about 10:00 daily. The control group was administered the same volume of vehicle. Body weight and food intake were measured daily during the experimental pe- 
riod.

Experiment 2: Fluvoxamine Administration to Food Restricted Rats At the beginning of the experiment, the rats were divided into two groups ( $n=6$ per group): a foodrestricted control (FRC) group, and a food-restricted and fluvoxamine-administered (FRF) group. Each group's food intake was restricted for $10 \mathrm{~d}$ to about $65 \%$ of the food intake before restriction. After 10 days' food restriction, rats were allowed to refeed for $10 \mathrm{~d}$ with ad libitum food access. They were administered the same volume of fluvoxamine or vehicle as that used in Experiment 1.

The body weight and food intake were measured daily during the experimental period in these studies. At the end of the experiment, rats were anesthetized using pentobarbital sodium (50 mg/kg, intraperitoneally (i.p.)). Blood was taken immediately from the carotid artery; then rats were perfused with saline, followed by $4 \%$ paraformaldehyde perfusion. After perfusion, the brain of each rat was removed from the skull immediately.

Hypothalamic Microdissection The brains were removed, cut into pieces, and immersed in 4\% paraformaldehyde overnight. They were stored for $2 \mathrm{~d}$ in $30 \%$ sucrose at $4{ }^{\circ} \mathrm{C}$, then frozen using dry ice and stored at $-80^{\circ} \mathrm{C}$ until analysis. Using a cryostatic microtome, $6-\mu \mathrm{m}$ slices were cut every $100 \mu \mathrm{m}$. They were then immunostained for NPY with polyclonal antisera raised in rabbits. The antigens were detected using the fluorescence technique.

Nonspecific background staining was blocked using blocking buffer, which contained 10\% normal donkey serum (Sigma-Aldrich Corp., U.S.A.), 1\% Triton X-100, and $0.1 \mathrm{M}$ phosphate buffered saline (PBS). The sections were incubated with rabbit anti-NPY antiserum (dilution 1:100; Sigma-Aldrich Corp., U.S.A.) overnight at $4{ }^{\circ} \mathrm{C}$. After washing in PBS, sections were incu-bated with $\mathrm{Cy} 3$-conjugated anti-rabbit immunoglobulin $\mathrm{G}$ (dilution 1:300; Jackson Immuno Research Laboratories, Inc., U.S.A.) overnight at $4{ }^{\circ} \mathrm{C}$. Primary antiserum was diluted in 5\% normal donkey serum, $0.3 \%$ Triton X-100, and $0.1 \mathrm{~m} \mathrm{PBS,} \mathrm{and} \mathrm{secondary} \mathrm{antiserum}$ was diluted in 5\% normal donkey serum, $0.1 \%$ Triton $\mathrm{X}-100$, and $0.1 \mathrm{M}$ PBS. The reaction was terminated after $1 \mathrm{~min}$ with three successive rinses in deionized water. The tissue sections were dried and mounted on cover glass. Using a conventional camera (CKX4; Olympus Optical Co., Ltd., Tokyo) and a CCD camera (Cool SNAP 5.0; Nippon Roper Scientific Co., Ltd., Tokyo), NPY immunostaining was examined. Hypothalamic NPY immunostaining was observed in ARC, PVN, and DMH.

Regional optical density of fluorescent immunostaining was transformed to a gray scale to evaluate NPY expression in selected brain regions. The digitized gray-scale image of each point was transformed automatically to a number from 0 (black) to 255 (white). The mean number was calculated in ARC, DMH, and PVN. Those numbers were subtracted from the mean value of each background area, as reported in a previous study. ${ }^{15}$

Statistical Analysis Data were analyzed using a twosample Student's $t$-test. They are presented herein as mean values \pm S.E. mean. Statistical significance of differences between the two groups was inferred for $p<0.05$.
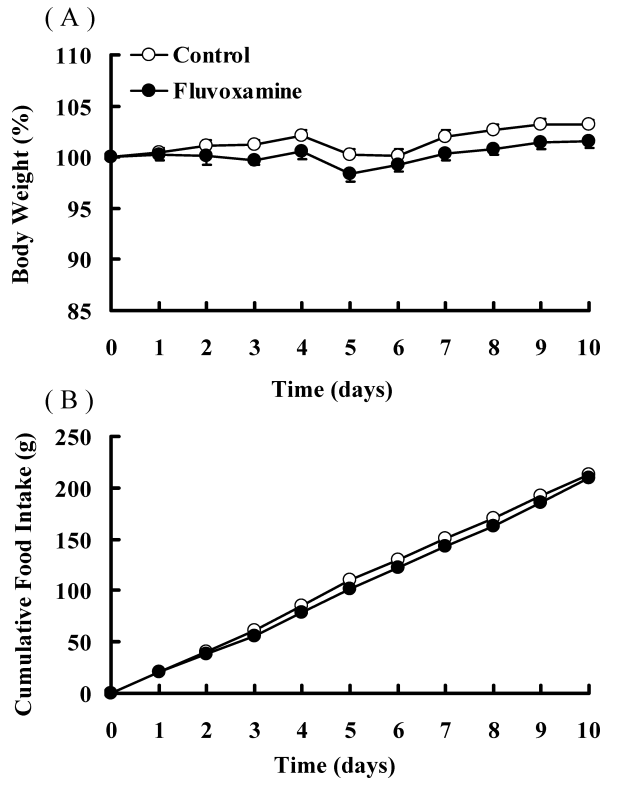

Fig. 1. Body Weight (A) and Cumulative Food Intake (B) Changes in Fluvoxamine $(100 \mathrm{mg} / \mathrm{kg})$-Administered and Vehicle-Administered ad libitum Fed Rats

Data are expressed as mean \pm S.E. mean. Because of the large standard deviation of the rat body weight ( $363-443 \mathrm{~g}$ ), we thought that a percentage display was appropriate.

\section{RESULTS}

Body Weight Change and Cumulative Food Intake in Fluvoxamine-Administered and Vehicle-Administered ad libitum Feeding Rats No significant difference was found in the body weight changes of rats receiving vehicle (Control) and those receiving fluvoxamine (Fluvoxamine; $103.2 \pm 0.2 \%$ in Control vs. $101.6 \pm 0.7 \%$ in Fluvoxamine; Fig. 1A). During early days of this study, fluvoxamine showed a tendency to decrease food intake. However, that tendency subsequently disappeared; eventually, no difference of cumulative food intake was observed between the two groups (213.2 $\pm 4.5 \mathrm{~g}$ in Control vs. 209.2 $\pm 3.1 \mathrm{~g}$ in Fluvoxamine; Fig. 1B).

Body Weight Change and Cumulative Food Intake in Fluvoxamine-Administered and Vehicle-Administered Food-Restricted Rats After 10 days' food restriction, both groups showed decreased body weight of about $10 \%$ from that before intervention (Fig. 2A). At the end of the refeeding period, the food-restricted and fluvoxamine-administered (FRF) group body weight was significantly lower than that of the food-restricted control (FRC) group $(101.3 \pm 0.5 \%$ in FRC vs. 97.2 $\pm 0.7 \%$ in FRF, $p<0.001)$. The cumulative food intake of FRF was significantly lower than that of FRC (209.7 $\pm 4.1 \mathrm{~g}$ in FRC vs. $183 \pm 3.5 \mathrm{~g}$ in FRF, $p<0.001$; Fig. 2B). On the first day of the refeeding period, both FRC and FRF rats showed higher levels of feeding than the respective levels of pre-food restriction, although the food intake of FRF was lower than that of FRC $(25.3 \pm 0.9 \mathrm{~g}$ in FRC vs. $22 \pm 0.7 \mathrm{~g}$ in FRF, $p<0.05$; Fig. 3).

Immunostaining Levels of Hypothalamic NPY We examined the levels of hypothalamic NPY immunostaining to determine the effects of fluvoxamine on hypothalamic NPY (Figs. 4, 5). In fact, FRF showed a tendency to reduce NPY immunostaining levels in the ARC compared to those of 
FRC, but the difference was not significant $(100 \pm 5.1 \%$ in FRC vs. $90 \pm 4 \%$ in FRF). In the DMH, the NPY immunostaining level of FRF was significantly lower than that of FRC
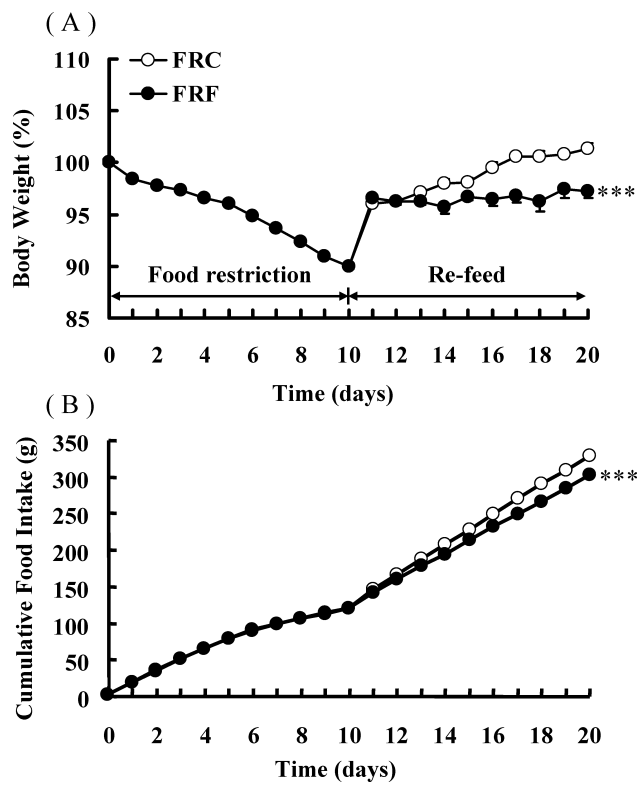

Fig. 2. Body Weight (A) and Cumulative Food Intake (B) Changes in Fluvoxamine $(100 \mathrm{mg} / \mathrm{kg})$-Administered and Vehicle-Administered FoodRestricted Rats

Data are expressed as mean \pm S.E. mean $(* * * p<0.001)$.
$(100 \pm 8 \%$ in FRC vs. $76 \pm 3.7 \%$ in FRF, $p<0.05)$.

The pattern of changes in NPY immunostaining levels in PVN induced by fluvoxamine resembled that found in the DMH. Fluvoxamine decreased PVN NPY levels significantly compared to the control $(100 \pm 5 \%$ in FRC $v s .84 .4 \pm 4.8 \%$ in FRF, $p<0.05)$.

\section{DISCUSSION}

Results of this study showed that the fluvoxamine did not affect appetite in normophagic middle-aged Wistar rats, although fluvoxamine appears to inhibit hyperphagia after a $10-\mathrm{d} 35 \%$ food restriction in the same rats. To the best of our knowledge, this is the first report describing a comparison

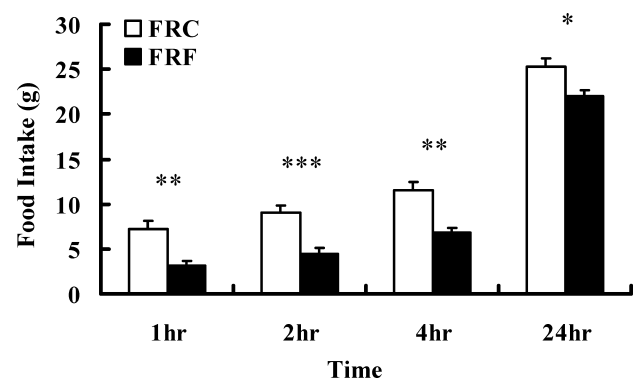

Fig. 3. Food Consumption during the Refeeding Period at the First Day and after 10 Days' Food Restriction

Data are expressed as mean \pm S.E. mean $(* p<0.05, * * p<0.01, * * * p<0.001)$.
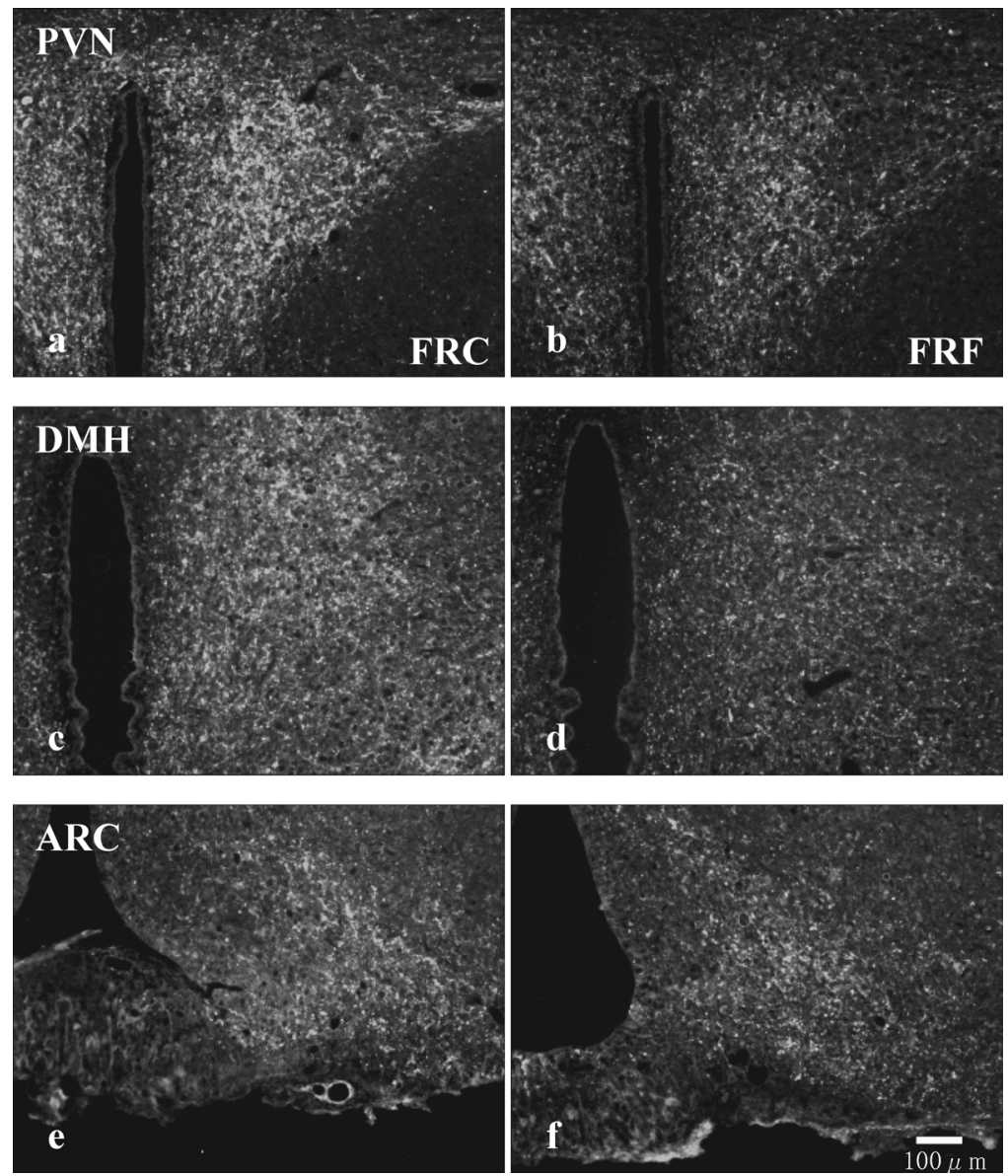

Fig. 4. Immunohistochemically Analyzed Sections of NPY in the Hypothalamus of Control (Left Panel) and Fluvoxamine-Treated Rats (Right Panel) Acronyms represent the following: PVN, paraventricular nucleus (a, b); DMH, dorsomedial hypothalamic nucleus (c, d); ARC, arcuate nucleus (e, f). 


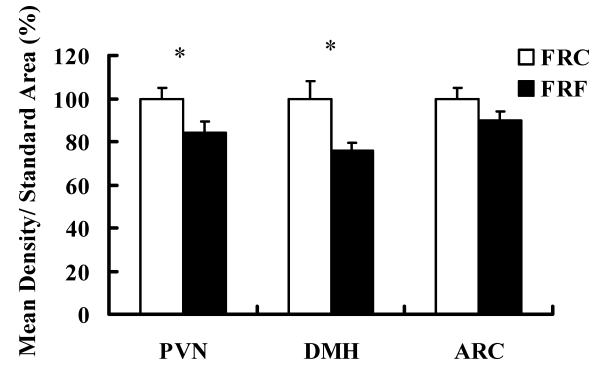

Fig. 5. Semiquantitative Analysis of NPY Immunostaining Levels in Hypothalamic Nuclei

Data are expressed as mean \pm S.E. mean $(* p<0.05)$.

of effects of fluvoxamine on the central appestat in normophagic and hyperphagic rats.

In Experiment 1, fluvoxamine was administered to normophagic middle-aged Wistar rats after one week of acclimation. Immediately after beginning fluvoxamine ingestion, the daily food intake showed a temporary decreasing tendency. However, fluvoxamine did not reduce the subsequent daily food intake or the cumulative food intake during the experimental period (Fig. 1B), nor was a significant difference found for body weight (Fig. 1A).

Actually, 5-HT is well known as an important substance to control eating behavior in the brain. Many agents that activate the 5-HT system in the brain, e.g., SSRI (fluvoxamine and fluoxetine), fenfluramine, dexfenfluramine, and sibutramine, are reportedly associated with anorexia. ${ }^{6,24-26)}$ Wieczorek et al. showed that fluvoxamine administration to young obese Zucker rats reduces their body weight and food consumption. $^{27)}$ These data conflict with results obtained in Experiment 1. Zucker rats are known to have a genetic alteration in the leptin receptor gene. They present a phenotype of hyperphagia. The dorsal raphe nucleus (DRN), projecting to the hypothalamus, is a major center of the 5-HT system; DRN has many leptin receptors in normal animals. ${ }^{28)}$ Zucker rats lack a leptin signal to DRN and might have a low 5-HT signal to the hypothalamus. Orexigenic neurons of NPY in the hypothalamus are negatively regulated by $5-\mathrm{HT}^{12)}$ For that reason, Zucker rats might have a low inhibitory signal to NPY neurons, which might engender hyperphagia. Fluvoxamine administration to obese Zucker rats might inhibit the orexigenic neuronal activity of NPY induced by increased 5HT in the hypothalamus.

Dryden et al. ${ }^{14)}$ also reported that fluoxetine administration to young Wistar rats decreases their food intake. This evidence conflicts with our data acquired for middle-aged Wistar rats. What underlies these differences between young and middle-aged Wistar rats? We examined those rats' appetite conditions to find likely explanations. Young rats used by Dryden et al. ${ }^{14)}$ might have been in a growth period: their weight increased about $23 \mathrm{~g}$ per week. The data suggest that approximately 9-week-old Wistar rats gained about $9 \%$ of their initial body weight. On the other hand, the middle-aged Wistar rats used in our study were apparently past their growth period: their weight gain was only $4 \mathrm{~g}$ per week $(1 \%$ gain of their body weight). Therefore, young rats might have been in a high-appetite condition because of their growth processes. Gruenewald et $a .^{29,30)}$ reported that the low basal NPY mRNA expression in the brain and the reduction of basal food intake are strongly correlated with aging. Furthermore, Akimoto-Takano et al. $^{31)}$ reported that aging attenuates the appetite-stimulating effect of NPY in the brain. Moreover, Gutierrez et al. ${ }^{15)}$ suggested that differences in the age and maturation of rats might affect the appetite-suppression effectiveness of SSRI. For those reasons, we consider that young Wistar rats used in the previous study reported by Dryden et al. ${ }^{14)}$ were in a hyperphagic condition. Consequently, the inhibitory effects of fluvoxamine on NPY synthesis and appetite behavior were remarkable in young Wistar rats compared to those in the normophagic middle-aged Wistar rats in our study.

The information presented above leads us to infer that the appetite level is an important determinant of the appetite control effect of fluvoxamine. Actually, Inoue et al. ${ }^{32)}$ reported for food-access-restricted hyperphagic rats that fluvoxamine suppressed food intake for $3 \mathrm{~h}$. We examined the effects of fluvoxamine administration in a model of middleaged Wistar rats that were hyperphagic because of food restriction (Experiment 2).

In Experiment 2, the food volume was restricted to about $65 \%$ of the food volume during the acclimation period; this intervention led to an approximately $10 \%$ weight loss for $10 \mathrm{~d}$. Middle-aged Wistar rats that were hyperphagic because of food restriction exhibited inhibitory effects of fluvoxamine in weight gain and food intake compared to vehicle-administered rats during refeeding (Fig. 2). To assess the mechanism of anorexia induced by fluvoxamine, NPY immunohistochemical analyses were performed in several hypothalamic areas; results showed reduction of NPY immunostaining levels in DMH and PVN induced by fluvoxamine administration during the recovery period.

Fluoxetine-induced hypophagia is reportedly accompanied by several changes in hypothalamic NPY expression. ${ }^{16)} \mathrm{A}$ decrease of NPY levels in PVN is reportedly induced by fluoxetine. ${ }^{14,15)}$ These data corresponded with our results, which suggest that fluvoxamine-induced anorexia in our study resulted from reduction of NPY immunostaining in PVN. Those mechanisms might resemble those of fluoxetine. Because PVN is a main region of terminal of NPY neurons from ARC and DMH, fluvoxamine might induce 5-HT reuptake inhibition and increase 5-HT concentration at either ARC or DMH, or both. These changes were shown to decrease NPY immunostaining levels in DMH and PVN in our study. Changes in NPY immunostaining were also observed in DMH in our study. In fact, 5-HT is transported specifically from DRN to DMH neurons, which synthesize NPY; this NPY neuron in DMH projects into PVN. ${ }^{13,20)}$ An important controlling factor of eating behavior is 5-HT, which is apparently mediated by the NPY system. Some evidence indicates that NPY neurons are negatively regulated by the 5-HT system in the hypothalamus. ${ }^{12,33,34)}$ Via central 5-HT ${ }_{1 \mathrm{~B}}$ receptors, 5-HT is known to suppress food intake and body weight gain. In addition, NPY and 5- $\mathrm{HT}_{1 \mathrm{~B}}$ receptor-immunopositive neurons co-exist in the hypothalamus. ${ }^{12,35)}$ Anorexigenic effects of some agents appear to mediate the $5-\mathrm{HT}_{1 \mathrm{~B}}$ receptor in the hypothalamus. ${ }^{423}$ This evidence suggests that the NPY content in DMH was decreased by the increase in 5-HT induced by fluvoxamine. Moreover, the decrease of NPY in PVN might be induced by a decrease of NPY delivery from the DMH. 
On the other hand, no significant effect of fluvoxamine was found on NPY immunostaining levels in ARC despite a trend toward a decrease in NPY. These data are supported by evidence reported by Gutierrez et al. ${ }^{15)}$ and Dryden et al. ${ }^{14)}$ for hyperphagic obese Zucker rats. Dryden et al. ${ }^{14)}$ reported conflicting evidence for young Wistar rats: fluoxetine decreased NPY in PVN, increased NPY in ARC, and did not affect DMH. These results are inconsistent with the results described by Gutierrez et al., ${ }^{15)}$ results of our previous study, and those for Dryden's Zucker rats. The changes in regional NPY levels induced by SSRI treatments might result from the appetite level, variation in volume or duration of the agent's administration, or strain differences. Many conflicting data have been reported related to the effect of SSRI on the content and secretion of NPY in various areas of the hypothalamus: further investigation is needed.

In conclusion, fluvoxamine administration to normophagic middle-aged Wistar rats decreased neither their weight nor food intake. However, fluvoxamine administration to food-restricted rats inhibited weight gain and food intake during the recovery period from a food-restricted condition in hyperphagic middle-aged Wistar rats. This result might be attributable to increased 5-HT in DMH, which might decrease NPY synthesis in the DMH-PVN neural network. Results suggest that the effective administration of fluvoxamine on appetite control is responsible for an appetite condition.

\section{REFERENCES}

1) Zimmet P., Magliano D., Matsuzawa Y., Alberti G., Shaw J., J. Atheroscler. Thromb., 12, 295-300 (2005).

2) Bays H. E., Obes. Res., 12, 1197-1211 (2004).

3) Li Z., Maglione M., Tu W., Mojica W., Arterburn D., Shugarman L. R., Hilton L., Suttorp M., Solomon V., Shekelle P. G., Morton S. C., Ann. Intern. Med., 142, 532-546 (2005).

4) Funahashi H., Takenoya F., Guan J. L., Kageyama H., Yada T., Shioda S., Anat. Sci. Int., 78, 123-138 (2003).

5) Schwartz M. W., Woods S. C., Porte D., Jr., Seeley R. J., Baskin D. G., Nature (London), 404, 661-671 (2000).

6) Guy-Grand B., Apfelbaum M., Crepaldi G., Gries A., Lefebvre P., Turner P., Lancet, 2, 1142-1145 (1989).

7) Mathus-Vliegen E. M., van de Voorde K., Kok A. M., Res A. M., J. Intern. Med., 232, 119-278 (1992).

8) Bray G. A., Blackburn G. L., Ferguson J. M., Greenway F. L., Jain A. K., Mendel C. M., Mendels J., Ryan D. H., Schwartz S. L., Scheinbaum M. L., Seaton T. B., Obes. Res., 7, 189-198 (1999).

9) Lames W. P., Astrup A., Finer N., Hilsted J., Kopelman P., Rössner S., Saris W. H., Van Gaal L.F., Lancet, 356, 2119-2125 (2000).

10) Leibowitz S. F., Weiss G. F., Suh J., Pharmacol. Biochem. Behav., 37,
$735-742$ (1990).

11) Guy J., Pelletier G., Bosler O., Neurosci. Lett., 85, 9-13 (1988).

12) Heisler L. K., Jobst E. E., Sutton G. M., Zhou L., Borok E., ThorntonJones Z., Liu H. Y., Zigman J. M., Balthasar N., Kishi T., Lee C. E., Aschkenasi C. J., Zhang C. Y., Yu J., Boss O., Mountjoy K. G., Clifton P. G., Lowell B. B., Friedman J. M., Horvath T., Butler A. A., Elmquist J. K., Cowley M. A., Neuron, 51, 239-249 (2006).

13) Vanhatalo S., Soinila S., Eur. J. Neurosci., 10, 1930-1935 (1998).

14) Dryden S., Frankish H. M., Wang Q., Pickavance L., Williams G., Neuroscience, 72, 557-566 (1996).

15) Gutierrez A., Saracibar G., Casis L., Echevarria E., Rodriguez V. M., Macarulla M. T., Abecia L. C., Portillo M. P., Obes. Res., 10, 532540 (2002).

16) Myung C. S., Kim B. T., Choi S. H., Song G. Y., Lee S. Y., Jahng J. W., Arch. Pharm. Res., 28, 716-721 (2005).

17) Allen Y. S., Adrian T. E., Allen J. M., Tatemoto K., Crow T. J., Bloom S. R., Polak J. M., Science, 221, 877-879 (1983).

18) Gray T. S., Morley J. E., Life Sci., 38, 389-401 (1986).

19) Bi S., Ladenheim E. E., Schwartz G. J., Moran T. H., Am. J. Physiol. Regul. Integr. Comp. Physiol., 28, R254-R260 (2001).

20) Kesterson R. A., Huszar D., Lynch C. A., Simerly R. B., Cone R. D., Mol. Endocrinol., 11, 630-637 (1997).

21) Stanley B. G., Daniel D. R., Chin A. S., Leibowitz S. F., Peptides, 6 1205-1211 (1985).

22) Bi S., Robinson B. M., Moran T. H., Am. J. Physiol. Regul. Integr Comp. Physiol., 285, R1030-R1036 (2003).

23) Shin M. S., Kim H., Chang H. K., Lee T. H., Jang M. H., Shin C., Lim B. V., Lee H. H., Kim Y. P., Yoon J. H., Jeong I. G., Kim C. J., Neurosci. Lett., 346, 157-160 (2003).

24) Jackson H. C., Needham A. M., Hutchins L. J., Mazurkiewicz S. E., Heal D. J., Br. J. Pharmacol., 121, 1758-1762 (1997).

25) Levin B. E., Dunn-Meynell A. A., Am. J. Physiol. Regul. Integr. Comp. Physiol., 279, R2222-R2228 (2000).

26) Lucas J. J., Yamamoto A., Scearce-Levie K., Saudou F., Hen R., J. Neurosci., 18, 5537-5544 (1998).

27) Wieczorek I., Schulz C., Jarry H., Lehnert H., Int. J. Obes. Relat. Metab. Disord., 25, 1566-1569 (2001).

28) Fernandez-Galaz M. C., Diano S., Horvath T. L., Garcia-Segura L. M., J. Neuroendocrinol., 14, 429-434 (2002).

29) Gruenewald D. A., Marck B. T., Matsumoto A. M., Endocrinology, 137, 4460-4467 (1996).

30) Gruenewald D. A., Naai M. A., Marck B. T., Matsumoto A. M., Endocrinology, 134, 2383-2389 (1994).

31) Akimoto-Takano S., Sakurai C., Kanai S., Hosoya H., Ohta M., Miyasaka K., Neuroendocrinology, 82, 256-263 (2005).

32) Inoue K., Kiriike N., Fujisaki Y., Kurioka M., Yamagami S., Physiol. Behav., 61, 603-608 (1997).

33) Makarenko I. G., Meguid M. M., Gatto L., Chen C., Ramos E. J. B., Goncalves C. G., Ugrumov M. V., Neurosci. Lett., 383, 322-327 (2005).

34) Makarenko I. G., Meguid M. M., Gatto L., Goncalves C. G., Ramos E. J. B., Chen C., Ugrumov M. V., Neurosci. Lett., 376, 71-75 (2005).

35) Makarenko I. G., Meguid M. M., Ugrumov M. V., Neurosci. Lett., 328, $155-159$ (2002). 